\title{
Omic analysis of the endangered Taxaceae species Pseudotaxus chienii revealed the differences in taxol biosynthesis pathway between Pseudotaxus and Taxus yunnanensis trees
}

Chunna $\mathrm{Yu}^{1,2}$, Chengchao Zhang ${ }^{1,2}$, Xinyun $\mathrm{Xu}^{1,2}$, Jiefang Huang ${ }^{1,2}$, Yueyue Chen ${ }^{1,2}$, Xiujun Luo ${ }^{1,2}$, Huizhong Wang ${ }^{1,2^{*}}$ and Chenjia Shen ${ }^{1,2^{*}}$ (D)

\begin{abstract}
Background: Taxol is an efficient anticancer drug accumulated in Taxus species. Pseudotaxus chienii is an important member of Taxaceae, however, the level of six taxoids in P. chienii is largely unknown.

Results: High accumulation of 10-DAB, taxol, and 7-E-PTX suggested that $P$. chienii is a good taxol-yielding species for large-scale cultivation. By the omics approaches, a total of 3,387 metabolites and 61,146 unigenes were detected and annotated. Compared with a representative Taxus tree (Taxus yunnanensis), most of the differentially accumulated metabolites and differential expressed genes were assigned into 10 primary and secondary metabolism pathways. Comparative analyses revealed the variations in the precursors and intermediate products of taxol biosynthesis between P. chienii and T. yunnanensis. Taxusin-like metabolites highly accumulated in P. chienii, suggesting a wider value of $P$. chienii in pharmaceutical industry.

Conclusions: In our study, the occurrence of taxoids in P. chienii was determined. The differential expression of key genes involved in the taxol biosynthesis pathway is the major cause of the differential accumulation of taxoids. Moreover, identification of a number of differentially expressed transcription factors provided more candidate regulators of taxol biosynthesis. Our study may help to reveal the differences between Pseudotaxus and Taxus trees, and promote resource utilization of the endangered and rarely studied P. chienii.
\end{abstract}

Keywords: Metabolome, Taxoids, Taxol biosynthesis pathway, Transcriptome, Resource utilization

\footnotetext{
* Correspondence: whz62@163.com; shencj@hznu.edu.cn

${ }^{1}$ College of Life and Environmental Sciences, Hangzhou Normal University, Hangzhou 310036, China

Full list of author information is available at the end of the article
}

C C The Author(s). 2021 Open Access This article is licensed under a Creative Commons Attribution 4.0 International License, which permits use, sharing, adaptation, distribution and reproduction in any medium or format, as long as you give appropriate credit to the original author(s) and the source, provide a link to the Creative Commons licence, and indicate if changes were made. The images or other third party material in this article are included in the article's Creative Commons licence, unless indicated otherwise in a credit line to the material. If material is not included in the article's Creative Commons licence and your intended use is not permitted by statutory regulation or exceeds the permitted use, you will need to obtain permission directly from the copyright holder. To view a copy of this licence, visit http://creativecommons.org/licenses/by/4.0/ The Creative Commons Public Domain Dedication waiver (http://creativecommons.org/publicdomain/zero/1.0/) applies to the data made available in this article, unless otherwise stated in a credit line to the data. 


\section{Background}

Taxol (generic name paclitaxel) and its related products have been widely adopted and approved for the effective treatments of various types of cancers including breast, lung, non-small cell lung cancers [1]. For years, the barks and twigs of Taxaceae plants, Taxus trees in particular, are the major source of natural product taxol and its derivatives $[2,3]$. As the market demand for taxol increases annually, the illegal logging continues [4]. Due to over-exploitation and human activities, the population size of Taxaceae is shrinking $[5,6]$.

Taxaceae is the smallest family of conifers, consisting of six genera, including two former genera of Cephalotaxaceae (Amentotaxus and Cephalotaxus) and four so called 'core' genera of Taxaceae (Austrotaxus, Pseudotaxus, Taxus and Torreya) [6,7]. Owing to the economic value of taxol, the study on Taxaceae trees has drawn numerous attentions in the past years [8].

Taxol is a classic representative of the more than 400 defined taxoids, which contain the unique taxane skeleton [9]. Not only taxol, but also some other taxoids have exhibited potent anti-tumor activities [10, 11]. To date, more and more compounds with the taxane core have been isolated and identified in various Taxaceae trees [12]. Four novel taxane derivatives with high anticancer potency were isolated from the ethanol extract of the whole plant of $T$. wallichiana [13]. Inhibitory effect of 13 taxane diterpenoids from $T$. chinensis on the proliferation of HeLa cervical cancer cells was evaluated and their chemical structures were deciphered [14]. Homoharringtonine, which is originally isolated from the genus Cephalotaxus, is another natural product in cancer chemotherapy $[15,16]$. However, information on the occurrence of taxol and other taxoids in P. chienii is very limited.

The previous studies have revealed three representative groups of taxoids, including 13-hydroxylated taxoids (baccatin III, paclitaxel, etc.), 14-hydroxylated taxoids (taxuyunnanin $\mathrm{C}$, yunnaxan, etc.), and $11(15 \rightarrow 1)$-abeo-taxoids [17]. As one of the most important 13-hydroxylated taxoids, Taxol's specific synthetic branch involves 19 steps from the diterpenoid progenitor geranylgeranyl diphosphate (GGPP), which is derived via the plastidial 2-C-methyl-Derythritol phosphate (MEP) pathway, to taxol [9]. To form the taxane skeleton, cyclization of GGPP to taxa-4(5),11(12)-diene is catalyzed by a slow-starter enzyme, taxadiene synthase (TS) [18]. Then, multiple modification enzymes, particularly from the CYP450, acyltransferase, aroyltransferase and benzoyltransferase families, participate in the structural modification of the skeleton [19-21]. A series of CYP450s, including the $2 \alpha-, 5 \alpha-, 7 \beta_{-}, \quad 9 \alpha-, \quad 10 \beta-$, and $13 \alpha-$ hydroxylases, are involved in the oxygenation steps of taxadiene at the $\mathrm{C}^{-2}, \mathrm{C}-5, \mathrm{C}-7, \mathrm{C}-9, \mathrm{C}-10$, and $\mathrm{C}$ 13 positions [22]. Meanwhile, several other transferases are considered to be involved in taxol biosynthesis, such as taxadien-5 $\alpha$-ol O-acetyl transferase (TAT), taxane-2 $\alpha$-O-benzoyltransferase $\quad$ (TBT), deacetylbaccatin III-10-O-acetyltransferase (DBAT), baccatin III-3-amino, 3-phenylpropanoyltransferase (BAPT), and $3{ }^{\prime}$-N-debenzoyl-2'-deoxytaxol- $N$-benzoyl transferase (DBTNBT) [23].

The taxol biosynthesis pathway is not a linear route but rather a complex network consisting of various divergent routes. For example, CYP450-mediated oxygenation steps engender a number of taxusin-like intermediate metabolites, such as $2 \alpha$-hydroxytaxusin and $7 \beta$-hydroxytaxusin [22]. As a 14-hydroxylated taxoid, Taxuyunnanin $\mathrm{C}$, has a significant effect on the enhancement of neuron growth factor activity, in cell suspension cultures of Taxus species [24, 25]. A 14 $\beta$ hydroxylase $(\mathrm{T} 14 \mathrm{OH})$ is involved in the diversion of the pathway towards 14 $\beta$-hydroxylated taxoids, such as taxuyunnanin C [26].

T. yunnanensis, an endangered and slow-growing tree, is mainly distributed in Yunnan Province of China [27]. Phytochemical studies have isolated and identified many taxoids and other chemical compounds with biological activities from the seeds and twigs of $T$. yunnanensis. For examples, three novel taxane diterpenoids, namely baccatin VIII, baccatin IX, and baccatin X, were isolated from an ethanolic extract of the twigs and leaves of T. yunnanensis [28]. A novel heteropolysaccharide (TMP70W) with in vitro antitumor activity was structural identified from T. yunnanensis [29]. P. chienii, the sole species in the monotypic genus of Pseudotaxus, is another endangered yew and mainly distributed in the South China [5]. Morphological analysis showed a high similarity between $P$. chienii and the species from Taxus, differing from each other notably in the color of aril: white in Pseudotaxus and red in Taxus [6]. However, the differential accumulations of taxoids between Pseudotaxus and Taxus are largely unknown.

Technical advances in large-scale identification of genes and metabolites have facilitated to investigate the variations between two close species [30, 31]. Recently, several transcriptomes and metabolomes of $T$. yunnanensis have been reported [32-34]. So far no omic data of $P$. chienii was available. In the present study, an integrated metabolomic and transcriptomic approach was employed to elucidate the differential accumulations of taxoids between $P$. chienii and $T$. yunnanensis and the underlying mechanisms. Our study may provide valuable information for the conservation and comprehensive utilization of these two endangered Taxaceae species. 


\section{Results}

Quantitative analysis determined the occurrence of taxol in $P$. chienii and the variations in taxoids between $P$. chienii and $T$. yunnanensis

To date, the occurrence of taxol in P. chienii is unknown. Herein, a target LC-MS approach was applied to check the occurrence of taxol and other taxoids, such as 7-E-DAP, 10-DAB, BAC, DAP, and 7-E-PTX, in P. chienii. Our data showed that all selected taxoids were detected, confirming the occurrence of taxoids in $P$. chienii (Fig. 1a). Quantitative analysis revealed the variations in taxoid contents between $P$. chienii and T. yunnanensis. In particular, 10-DAB, PTX, and 7-E-PTX highly accumulated in $P$. chienii. No significant differences in the contents of BAC, DAP, and 7-E-DAP were obeserved between P. chienii and T. yunnanensis (Fig. 1b).

\section{Overview of the metabolites}

The untargeted metabolomic analysis identified 3387 metabolites with annotations (Additional file 1). Three quality checking parameters, including total ion chromatograms, $m / z$ widths and retention time widths, were tested, indicating that the data generated a high degree of overlap and the UPLC-MS/MS analysis reached the required standards (Additional file 2). A PCA showed that the percentages of the explained values of PC1 and PC2 were 42.12 and $18.63 \%$, respectively, indicating a clear separation of the metabolomes from P. chienii and T. yunnanensis (Additional file 3). The metabolite profiling of the two Taxaceae species revealed great variations in their metabolomes (Fig. 2a).

According to their annotations, many metabolites were assigned into different metabolic pathways. KEGG enrichment analysis showed that most metabolites were belonged to amino acid metabolism, carbohydrate metabolism, terpenoids and polyketides metabolism, and cofactors and vitamins metabolism (Fig. 2b). HMDB
Super Class analysis showed that 311 metabolites were grouped to 11 major categories, such as "organic acids and derivatives" (161 metabolites), "organoheterocyclic compounds" (31 metabolites), "organooxygen compounds" (24 metabolites), "phenylpropanoids and polyketides" (23 metabolites), and "nucleosides, nucleotides, and analogue" (17 metabolites) (Fig. 2c).

\section{Overview of the transcriptome}

Using similar samples, RNA-sequencing yielded 50.35 $\mathrm{Gb}$ of sequence data, including $25.40 \mathrm{~Gb}$ from $P$. chienii and $24.95 \mathrm{~Gb}$ from T. yunnanensis (Additional file 4). The clean reads were assembled and produced 133,507 transcripts (N50: 1561), with a mean length of $513 \mathrm{bp}$, and 61,146 unigenes (N50: 1606), with a mean length of $419 \mathrm{bp}$ (Additional file 5). Analysis of size distribution of all transcripts showed that $11.48 \%$ of the transcripts $11.01 \%$ of the unigenes were $>2000 \mathrm{bp}$ in length (Additional file 5). For annoatation, 61,146 unigenes were annotated by several common databases (Additional file 5). The species distribution suggested that the majority of the unigenes displayed significant similarities to known proteins from Picea sitchensis, Amborella trichopoda, and Quercus suber (Additional file 5).

Analysis of DEGs showed 4,215 T. yunnanensis highlyexpressed unigenes and 4,845 P. chienii highly-expressed unigenes (Fig. 3a). Most of the DEGs were assigned into different GO terms belonging to three major categories (Fig. 3b and Additional file 6). KEGG analysis showed that 34 KEGG pathways were significantly enriched in the DEGs between T. yunnanensis and P. chienii (Additional file 7). The top 20 enriched KEGG pathways, such as the 'phenylpropanoid biosynthesis', 'plant-pathogen interaction', and 'plant hormone signal transduction' pathways, were shown (Fig. 3c).
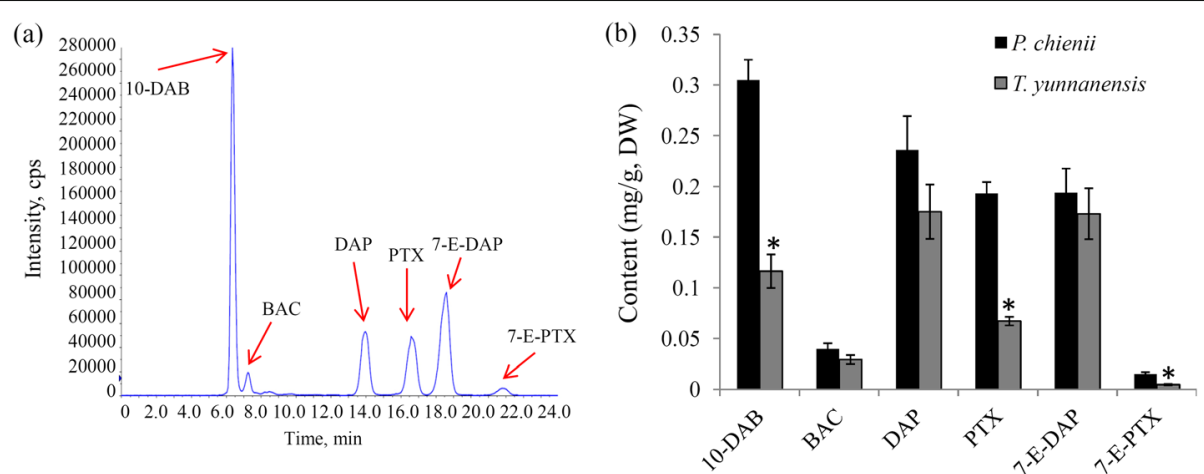

Fig. 1 Determination of the contents of taxoids in P. chienii and T. yunnanensis. a A representative TIC chromatogram of six taxoids. 10-DAB: 10deacetylbaccatin III; BAC: baccatin III; DAP: 10-deacetylpaclitaxel; PTX: paclitaxel; 7-E-DAP: 7-epi 10-desacetyl paclitaxel; 7-E-PTX: 7-epipaclitaxel. b The contents of six taxoids in $P$. chienii and $T$. yunnanensis. A $P$ value $<0.01$ was considered to be statistically significant and indicated by ${ }^{\prime *}$ 

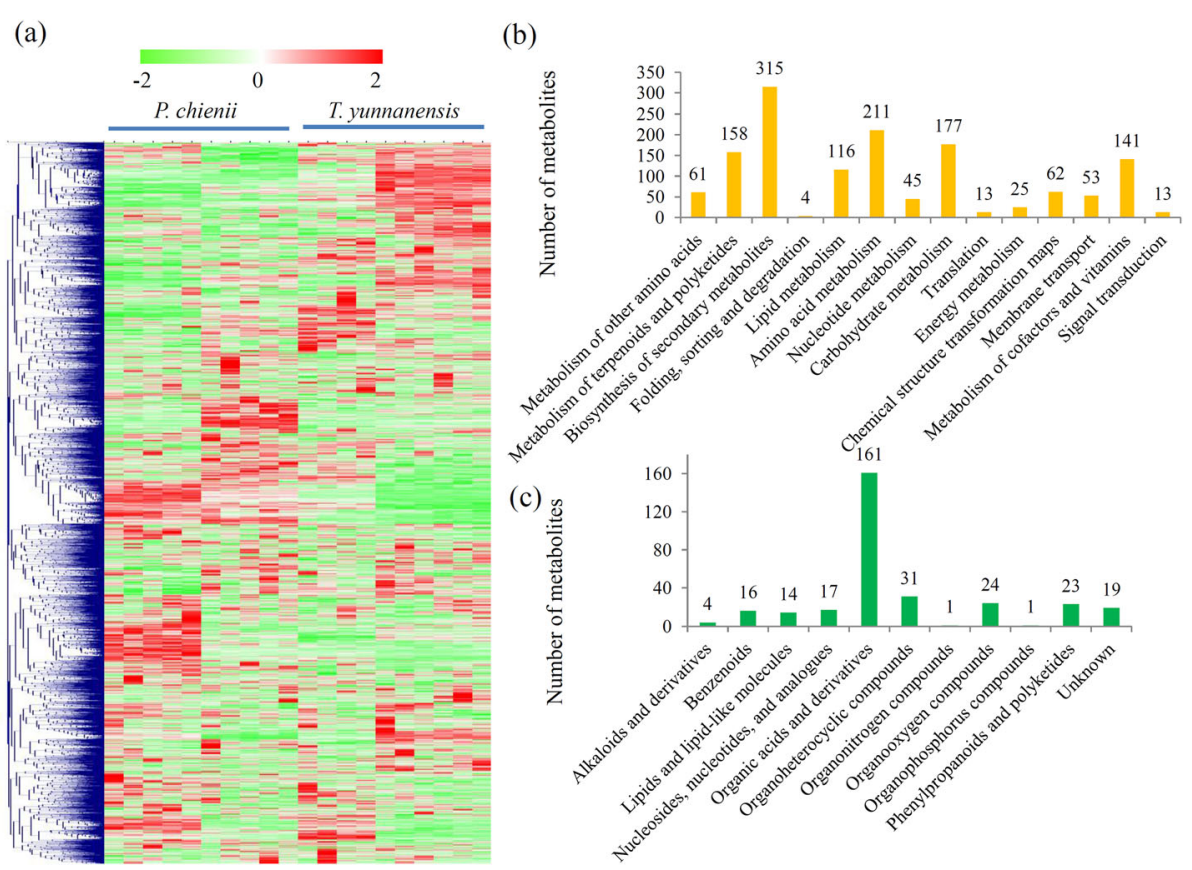

Fig. 2 The variations in the abundance of metabolites between $P$. chienii and T. yunnanensis. a A heatmap of the metabolites identified in the metabolomes of $P$. chienii and $T$. yunnanensis $(N=10)$. The heatmap scale ranges from -2 to +2 on a $\log _{2}$ scale. $\mathbf{b}$ KEGG analysis of all the identified metabolites. c HMDB Super Class analysis of all the identified metabolites

(a)

(d)

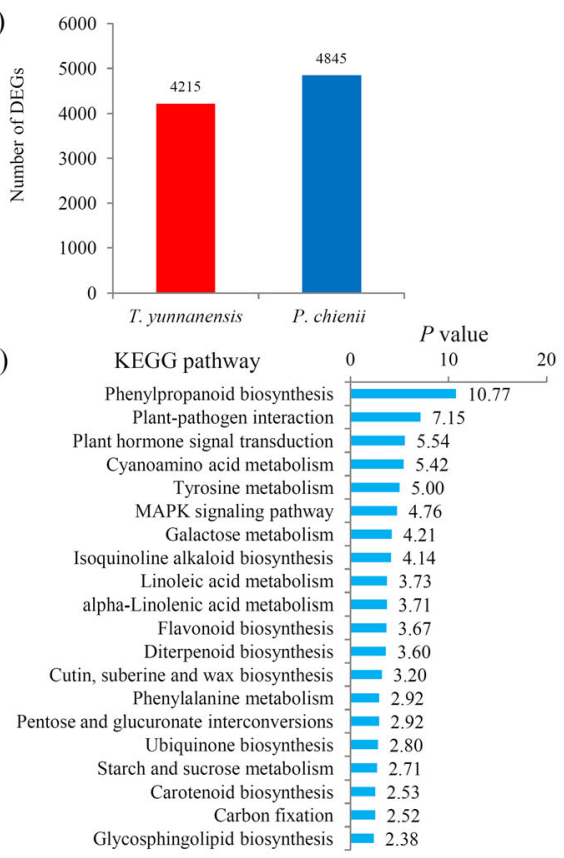

(c)

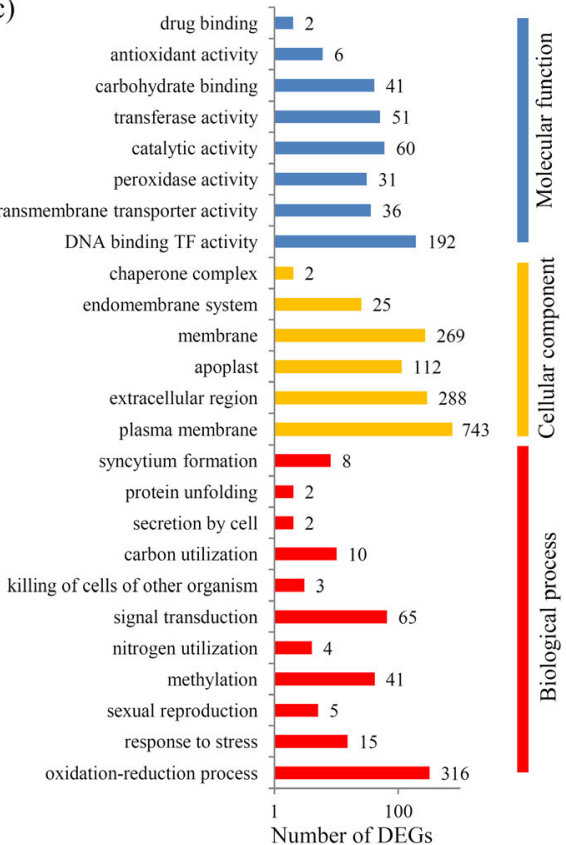

Fig. 3 Identification of the DEGs between $P$. chienii and T. yunnanensis. a The numbers of $P$. chienii predominantly expressed genes and T. yunnanensis predominantly expressed genes. $\mathbf{b} \mathrm{GO}$ analysis of all the DEGs between $P$. chienii and $T$. yunnanensis. $\mathbf{c}$ KEGG analysis of all the DEGs between $P$. chienii and $T$. yunnanensis 
Variations in primary and secondary metabolism between $T$. yunnanensis and $P$. chienii

According to their annotations, a large number of DEGs were involved in the primay and secondary metabolism, and the majority of the DEGs were grouped into 46 KEGG terms belonging to 11 major categories. Significance values of each KEGG term were calculated and shown in Additional file 8. In detail, two alkaloid-related pathways, five amino acid-related pathways, two flavonoid-related pathways, one hormone-related pathway, three lipid-related pathways, one phenylpropanoidrelated pathway, all three pigment/vitamin pathways, three saccharide-related pathways, one terpenoid-related pathway, and one ubiquinone-related pathway, showed significant differences between $T$. yunnanensis and $P$. chienii (Fig. 4a).

Untargeted metabolomic analysis identified 313 differentially accumulated metabolites (DAMs), 129 DAMs of which were assigned into different primary and secondary metabolite categories (Fig. 4b). The numbers of DAMs belonging to each category were shown in Fig. 4c.

Variations in the precursors of taxol biosynthesis between $T$. yunnanensis and $P$. chienii

The MEP pathway provided a key precursor, GGPP, for taxol biosynthesis [35]. Based on the sequence similarity to model plants, a predicted MEP pathway is showed in Fig. 5a. Our transcriptome data revealed at least one unigene encoding one enzyme that is involved in the MEP pathway (Additional file 9). In the MEP pathway, three DXS encoding unigenes, one DXR encoding unigene, one MCT encoding unigene, one CMK encoding unigene, two MDS encoding unigene, two HDS encoding unigenes, one HDR encoding unigene, one GGPS encoding unigene, and three GGPPS encoding unigenes, were identified. Most of the MEP pathway-related genes highly expressed in T. yunanensis, except for $C M K$, GGPPS1 and GGPPS2 (Fig. 5b).

Furthermore, our metabolome data identified four intermediate metabolites of the MEP pathway, including 2-C-methyl-D-erythritol 4-phosphate, 2-C-methyl-Deryhritol 2,4-cyclodiphosphate, geranyl diphosphate (GPP), and GGPP. Among these intermediate products, 2-C-methyl-D-eryhritol 2,4-cyclodiphosphate, GPP, and GGPP highly accumulated in T. yunnanensis (Fig. 5c).

\section{Variations in the taxol biosynthesis pathway between $T$. yunnanensis and $P$. chienii}

Taxol biosynthesis involves a complicated metabolic pathway consisting of several intermediate products and their catalyzing enzymes [9]. In our study, 20 unigenes encoding nine key enzymes involved in the taxol biosynthesis pathway were identified, including one TS
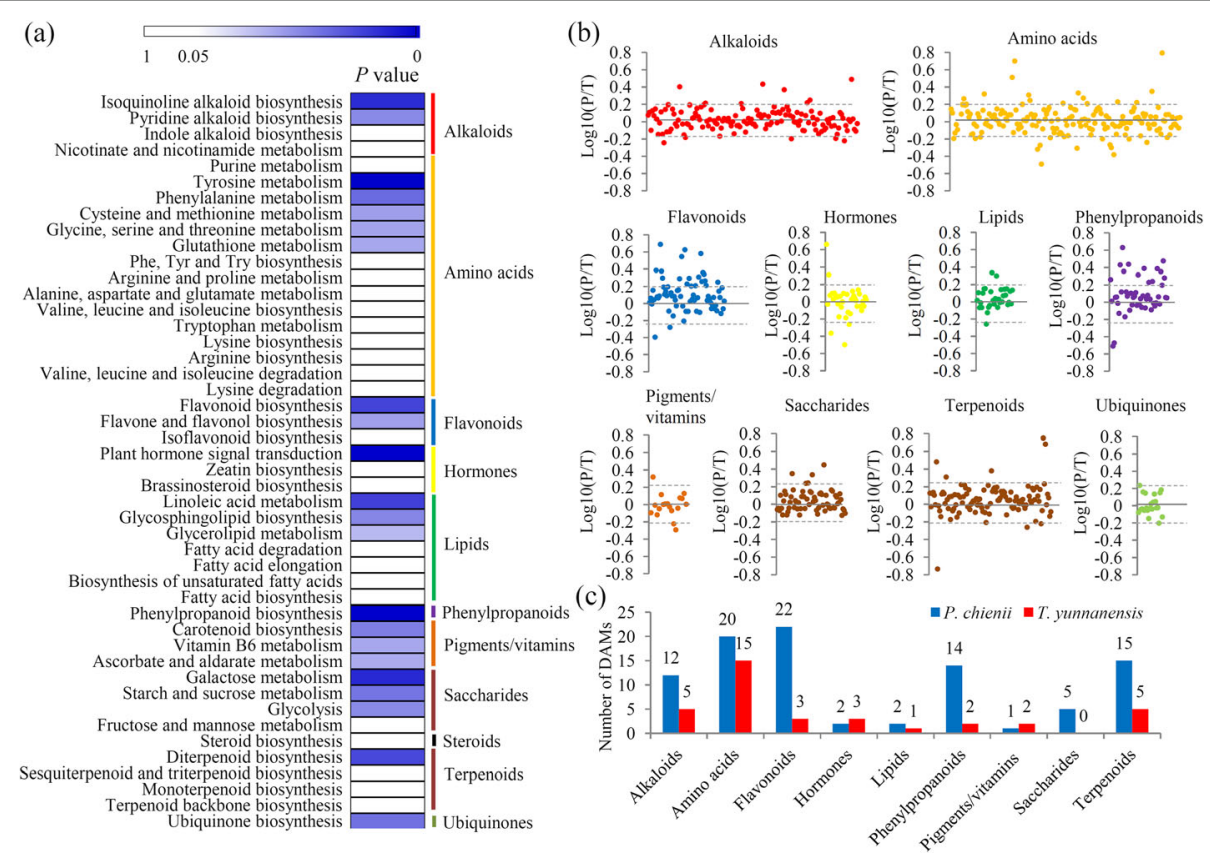

Fig. 4 Comparative analysis of DEGs and DAMs between $P$. chienii and T. yunnanensis. a KEGG enrichment analysis of the DEGs. The significant $P$ value of each KEGG term between $P$. chienii and T. yunnanensis was shown by a heatmap. All the KEGG terms were grouped into 11 metabolismrelated categories, which were indicated by different color bars. $\mathbf{b}$ The relative abundances of the metabolites belonging to various major metabolic categories. c The numbers of $P$. chienii predominantly accumulated and $T$. yunnanensis predominantly accumulated metabolites in different metabolic categories 

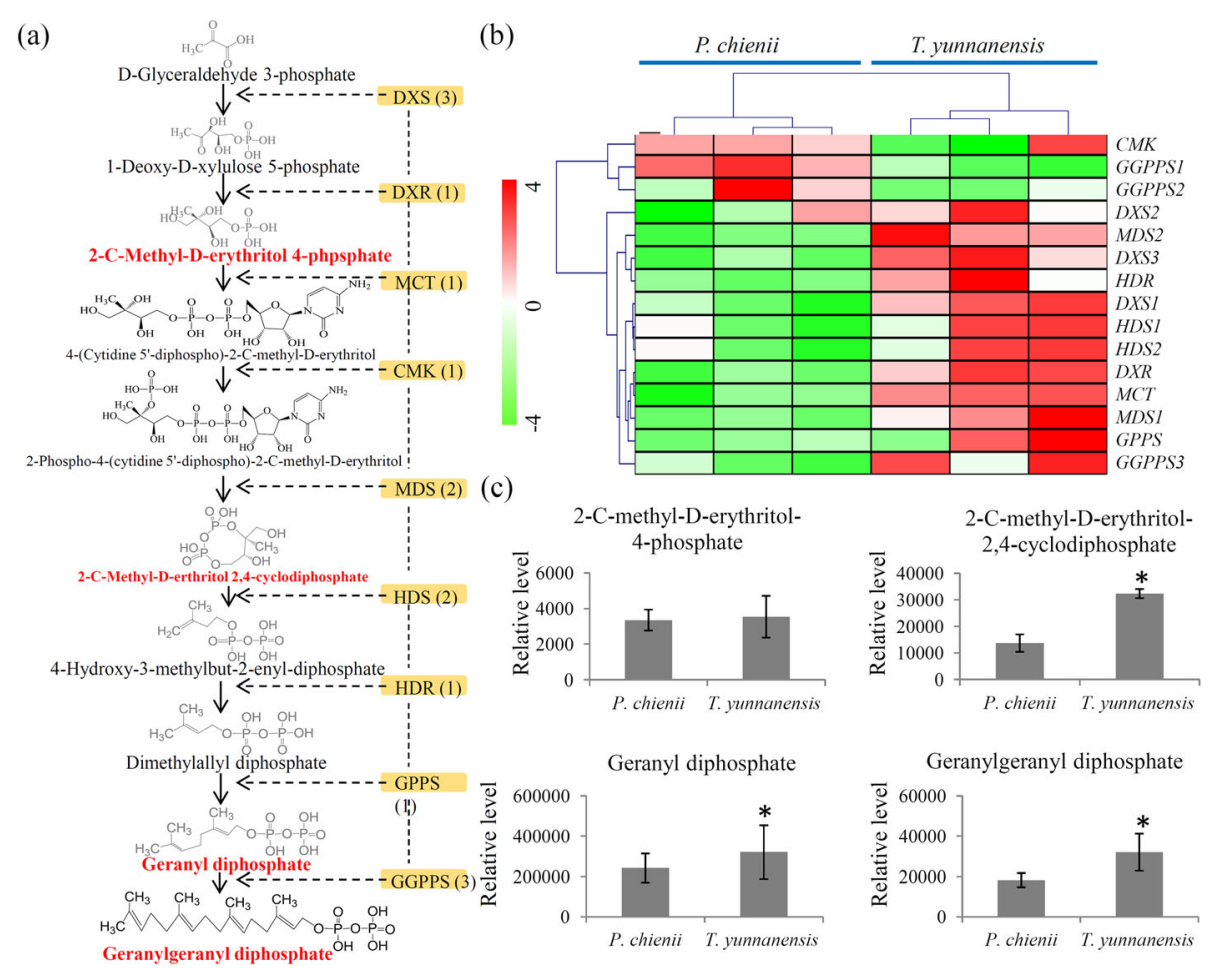

Fig. 5 Integrated metabolomic and transcriptomic analysis of the MEP pathway. a Overview of the MEP pathway. The orange backgrounds indicated the genes identified by the transcriptome and red font indicated the metabolites identified by the metabolome. $\mathbf{b}$ Differential expression of the key genes involved in the MEP pathway. The heatmap scale ranges from -4 to +4 on a $\log _{2}$ scale. $\mathbf{c}$ Differential accumulation of the intermediate metabolites involved in the MEP pathway. "**" represents significant differences $(P<0.05)$

encoding gene, four $\mathrm{T} 5 \alpha \mathrm{H}$ encoding genes, three TAT encoding genes, two $\mathrm{T} 13 \alpha \mathrm{H}$ encoding genes, five $\mathrm{T} 10 \beta \mathrm{H}$ encoding genes, one TBT encoding gene, one DBTNBT encoding gene, one DBAT encoding gene and two BAPT encoding genes (Fig. 6a and Additional file 10). The $B A P T 1 / 2, D B A T, T 5 \alpha H 1 / 3, T 10 \beta H 1 / 2 / 3$ genes highly expressed in $P$. chienii and the TAT1/2/3, DBTNBT,

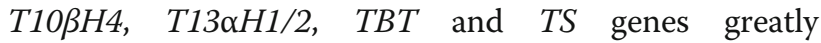
expressed in T. yunnanensis (Fig. 6b).

Our metabolome data identified six intermediate metabolites involved in taxol biosynthesis. Among these intermediate metabolites, 10-deacetyl-2-debenzoylbaccatin, and 10-deacetylbaccatin highly accumulated in $P$. chienii and 10 $\beta, 14 \beta$-dihydroxytaxa-4(20),11(12)-dien-5 $\alpha$ yl acetate and 3 '- $N$-debenzoyltaxol highly accumulated in T. yunnanensis (Fig. 6c).

\section{Variations in dead-end metabolites of taxol biosynthesis} and 14-hydroxylated taxoids between $T$. yunnanensis and P. chienii

Our transcriptome identified the encoding genes of $\mathrm{T} 2 \alpha \mathrm{H}$ and $\mathrm{T} 7 \beta \mathrm{H}$ that are involved in the metabolism of taxusin-like metabolites and the encoding gene of $\mathrm{T} 14 \mathrm{\beta H}$ that is involved in the biosynthesis of taxuyunnanin $\mathrm{C}$, a classic 14-hydroxylated taxoid (Fig. 7a and b). The $T 2 \alpha H$ gene highly expressed in P. chienii, while $T 7 \beta H$ and $T 14 \beta H$ genes predominantly expressed in T. yunnanensis (Fig. 7c). Furthermore, several deadend metabolites, such as (+)-taxusin, $2 \alpha$-hydroxytaxusin, $7 \beta$-hydroxytaxusin and $2 \alpha, 7 \beta$-dihydroxytaxusin, and one 14-hydroxylated taxoid, taxuyunnanin $\mathrm{C}$, were identified by the metabolomic analysis. The results showed that $(+)$-taxusin, $2 \alpha$-hydroxytaxusin, and $7 \beta-$ hydroxytaxusin highly accumulated in $P$. chienii. No significant differences in the levels of $2 \alpha, 7 \beta-$ dihydroxytaxusin and taxuyunnanin $C$ between $P$. chienii and T. yunnanensis were obersved (Fig. 7d).

\section{Expression validation of the key genes involved in the taxol pathway}

To investigate the differences in expression levels of the key genes involved in the taxol pathway, the relative levels of eight randomly selected taxol pathway-related genes were determined by qRT-PCR analysis. TS, DBTNBT, TAT, T13OH, T5OH genes were highly expressing in T. yunnanensis and TBT, BAPT, T10OH genes highly expressing in $P$. chienii (Fig. 8). 
(a)

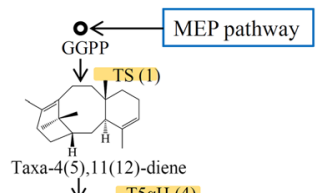

Taxa-4(5),11(12)-diene

$\downarrow$

Taxa-4(20), 11(12)-dien-5a-ol $\downarrow$ TAT (3) T13aH (2 Taxa-4(20) $11(12)$ $\downarrow$ TAT (3) T10ßH (5)

Taxa-4(20),11(12 4(20),11(12)-dien-5a-yl acetate dien-5 $\alpha, 13 \alpha$-diol

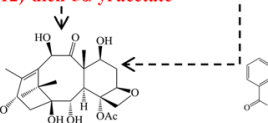
10-Deacetyl-2-debenzoylbaccatin III $\downarrow$ TBT (1)

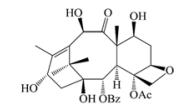

10-Deacetylbaccatin III $\downarrow$ DBAT (1)

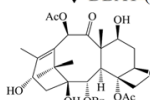

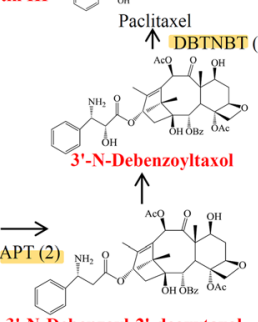

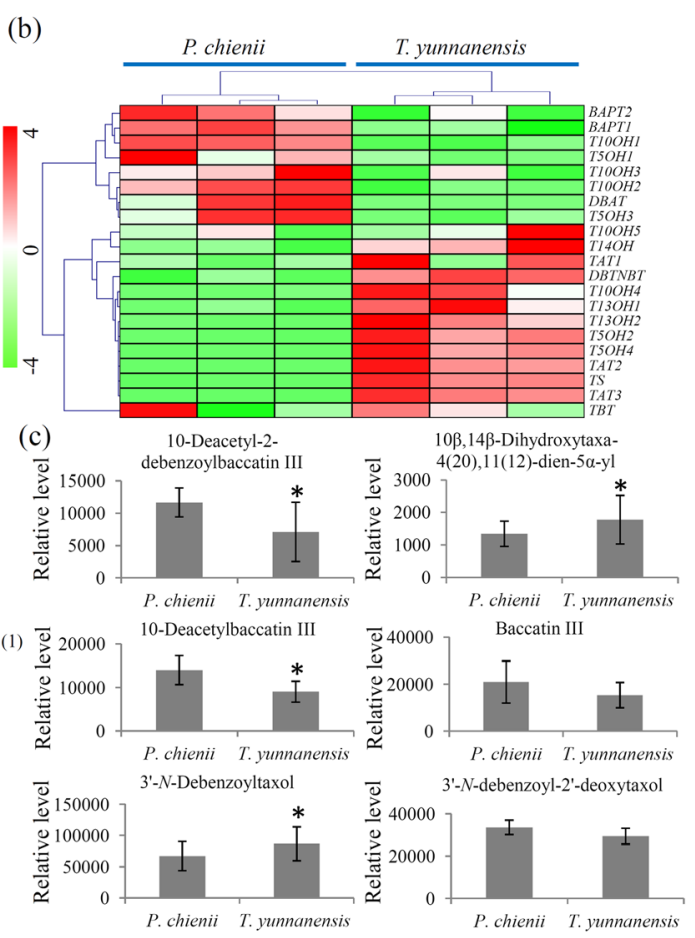

Fig. 6 Integrated metabolomic and transcriptomic analysis of the taxol biosynthesis pathway. a Overview of the taxol biosynthesis pathway. The orange backgrounds indicated the genes identified by the transcriptome and the red fonts indicated the metabolites identified by the metabolome. $\mathbf{b}$ Differential expression of the key genes involved in the taxol biosynthesis pathway. The heatmap scale ranges from -4 to +4 on a $\log _{2}$ scale. c Differential accumulation of the intermediate metabolites involved in the taxol biosynthesis pathway. " $*$ " represents significant differences $(P<0.05)$

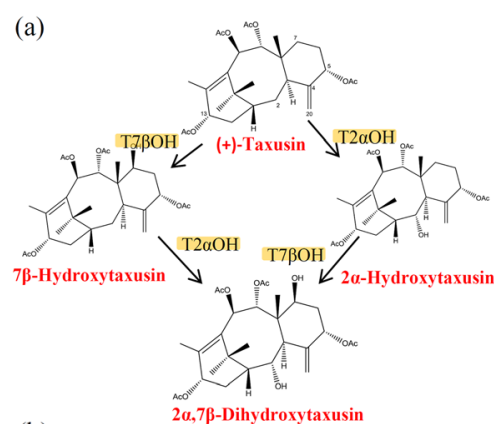

(b)

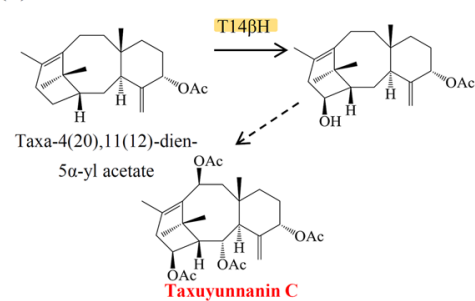

(c)

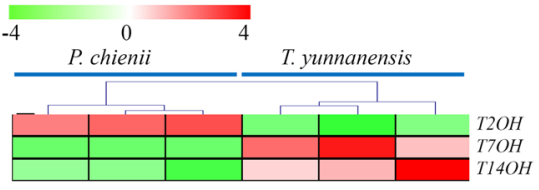

(d)
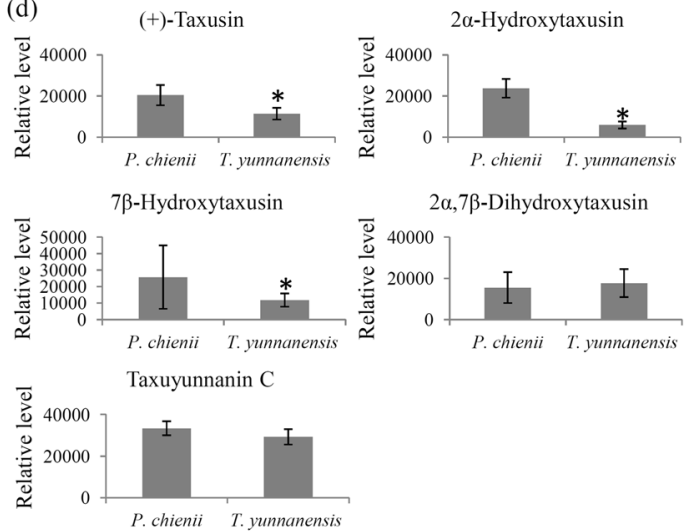

Fig. 7 Integrated metabolic and transcriptomic analysis of the branch of taxol biosynthesis pathway. The overview of the taxusin metabolism (a) and taxuyunnanin C metabolism (b) pathway. Orange background indicated the genes identified by the transcriptome and red font indicated the metabolites identified by the metabolome. (c) Differential expressed key genes involved in the branch of taxol biosynthesis pathway. The heatmap scale ranges from -4 to +4 on a $\log _{2}$ scale. (d) Differential accumulation of the metabolites involved in the branch of taxol biosynthesis pathway. " ${ }^{* \prime \prime}$ represents significant differences $(P<0.05)$ 

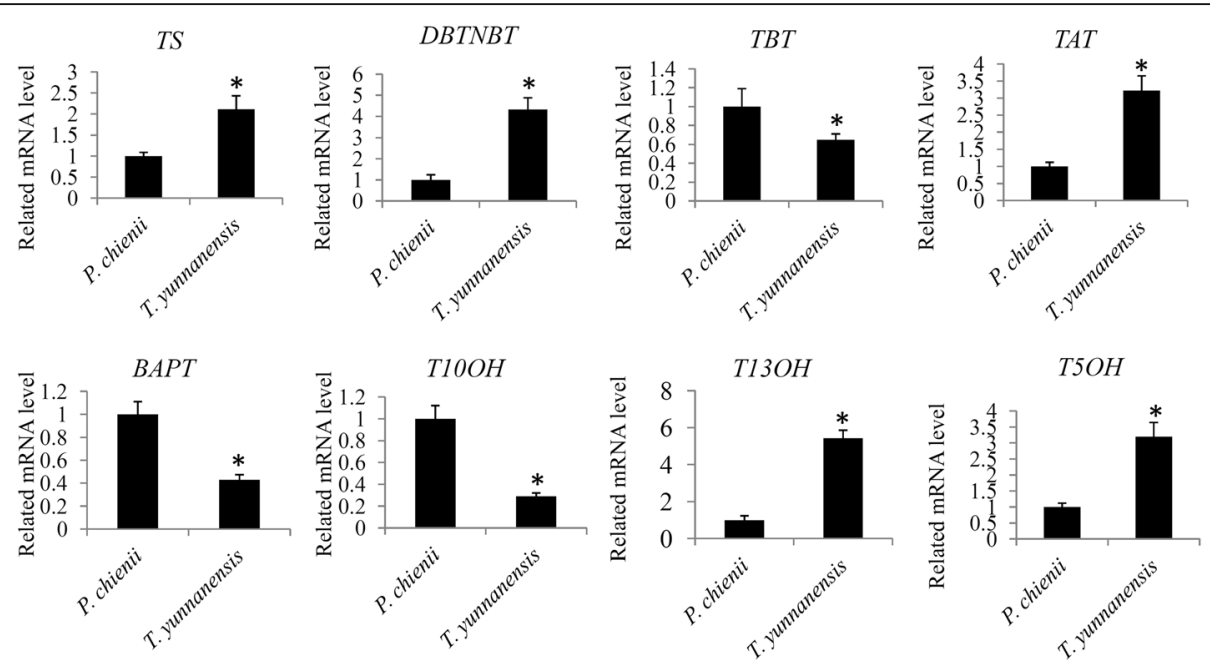

Fig. 8 Expression validation of the key genes involved in the taxol pathway. The significant variations $(P<0.05)$ are indicated by ${ }^{\prime * \prime}$ and error bars represent mean $\pm \mathrm{SD}(N=3)$

\section{Discussion}

To date, Taxus plants are the sole natural resource for taxol extraction, limiting the supplyment of taxol [36]. Huge economic values of taxol make the selection of high taxol-yielding Taxaceae species a topical research area $[2,32,33]$. Our data confirmed the occurence of taxol and other taxoids in P. chienii for the first time, suggesting that taxol is not the exclusive metabolite of Taxus genus. Further analysis showed that $P$. chienii contains a higher level of taxol than T. yunnanensis, indicating $P$. chienii a good taxol-yielding species for large-scale cultivation. Taking T. yunnanensis as a representative Taxus tree, an integrated metabolomic and transcriptomic approach was employed to elucidate the species-specific accumulations of taxoids between $P$. chienii and Taxus trees.

As the first transcriptome of $P$. chienii, our data provided 61,146 unigenes with full length or partial sequences, which is an important foundation for the investigation of the taxol biosynthesis pathway in P. chienii. Based on the unigene pool, we predicted the MEP pathway in P. chienii, which supplies a key precursor for the diterpenoid taxane core [35] (Fig. 5a). According to the metabolomes, GGPP was detected in both P. chienii and T. yunnanensis. In addition, an outline of taxol biosynthesis pathway, including 20 unigenes encoding nine key enyzmes, was also predicted (Fig. 6a). In the taxol biosynthesis pathway, six essential intermediates were identified in P. chienii. Our data suggested the presence of a classic taxol biosynthesis pathway in $P$. chienii.

The MEP pathway supplies three units of IPP and one unit of DMAPP to synthesize the diterpenoid taxane core [37]. The up-regulation of the MEP pathway was considered to have a positive effect on the precursor supply [38]. For example, DXS and DXR have been implicated as catalyzing slow steps in the biosynthesis of plastid-derived terpenoids [39]. GGPPS is another important enzyme that plays a regulatory role in taxoid production [40, 41]. Compared to T. yunnanensis, the expression levels of three DXS genes, one DXR gene and one GGPPS gene were relatively lower in P. chienii (Fig. $5 \mathrm{~b})$. The metabolomes confirmed that two major terminal products of the MEP pathway, GPP and GGPP, highly accumulated in T. yunnanensis, suggesting a more abundant precursor supply in T. yunnanensis.

In addition to taxol, several important imtermediate products of taxol biosynthesis also showed differential accumulations between $P$. chienii and T. yunnanensis. TS, catalyzing the formation of the intermediate product taxa-4(5),11(12)-diene, is a rate-limiting enzyme in the taxol biosynthesis pathway $[18,42]$. TAT catalyzes an important step of taxol biosynthesis, which is responsible for the acetylation of taxa-4(20),11(12)-dien-5 $\alpha$-ol [43]. In our study, one unigene encoding TS and three unigenes encoding TAT were identified, and they predominantly expressed in T. yunnanensis. DBAT, another rate-limiting enzyme, catalyzes the formation of baccatin III from 10-deacetylbaccatin III [44]. BAPT is responsible for the transfer of a C13-side chain to baccatin III [45]. Here, one unigene encoding DBAT and two unigenes encoding BAPT were identified, and they predominantly expressed in $P$. chienii. In pharmaceuticals industry, baccatin III and 10-DAB are key starting material of pacitaxel [46]. Our data showed that baccatin III and 10-DAB highly accumulated in P. chienii, suggesting a great economic value of $P$. chienii extracts. The differential expression levels of these key genes provided a possible explanation for the differential accumulation of 
the intermatdite products of taxol biosynthesis between T. yunnanensis and P. chienii.

The presence of dead-end metabolites and 14hydroxylated taxoids in Taxaceae trees has been previously reported [17]. For example, $(+)$-taxusin, a dead-end metabolite divergent from taxol formation, is a prominent metabolite of yew heartwood [47]. Taxusinlike metabolites display great anti-inflammatory and antinociceptive activities and are alternate substrates for testing microsomal oxygenase activities [47]. Interestingly, (+)-taxusin and its hydroxylated products highly accumulated in $P$. chienii, suggesting a wider value of $P$. chienii in pharmaceutical industry.

Recently, a large number of TFs have been considered to be involved in the regulation of taxol biosynthesis [48]. For examples, TcJAMYC1/2/3, TcERF12/15, and TcWRKY1/8/47 regulate the expression of paclitaxel biosynthetic genes by binding to their promoters [4951]. In our study, 12 differentially expressed bHLH genes, 24 differentially expressed ERF genes, and 16 differentially expressed WRKY genes were identified, providing more candidate regulators for the taxol biosynthesis. The MYC TFs are key regulators of the jasmonic acid (JA) signaling pathway and MeJA can improve the production of taxol [52]. In T. media, a number of biosynthesis gene promoters could be activated by MYC2, MYC3 and MYC4 [53]. In our study, 3 of 4 MYC TFs showed differential expressions, indicating potential differences in MYC-mediated JA signaling transduction between P. chienii and T. yunnanensis [54]. Differenially expressed MYC TFs might play an important role in taxol biosynthesis. Interestingly, a phloemspecific TmMYB3 was reported to be involved in the transcriptional regulation of paclitaxel biosynthesis [55]. Recently, expression profiling and posttranscriptional regulation of the R2R3-MYB TF family in $T$. chinensis have been well analyzed [56]. In our study, a number of MYB genes were identified, suggesting potential tissuespecific accumulation of taxoids in both $P$. chienii and $T$. yunnanensis.

\section{Conclusions}

In summary, the occurrence of taxol and other taxoids in $P$. chienii was confirmed by a UPLC-MS/MS method. Several taxoids, such as 10-DAB, taxol, and 7-E-PTX, highly accumulated in $P$. chienii, suggesting that it is a good taxol-yielding species for large-scale cultivation. Comparative metabolomic and transcriptomic analyses revealed the variations in the precursors, intermediate products and dead-end metabolites of taxol biosynthesis between $P$. chienii and T. yunnanensis. Furthermore, a number of differentially expressed TFs between $P$. chienii and T. yunnanensis were also identified, providing novel candidate regulators of taxol biosynthesis. Our study may aid in better understanding of the differences between Pseudotaxus and Taxus trees, and promoting comprehensive resource utilization of $P$. chienii, an endangered and rarely studied Taxaceae tree.

\section{Methods \\ Plant materials and sampling}

Cultivated 5-year-old P. chienii and T. yunnanensis trees were planted in a greenhouse at the campus of Hangzhou Normal University, Hangzhou, China, with a light/dark cycle of $12 / 12 \mathrm{~h}$ and $60 \% \sim 70 \%$ relative humidity at temperature of $25 \pm 1{ }^{\circ} \mathrm{C}$. The authorities responsible for the Taxus resources are the Mount Tianmu National Nature Reserve, who provided permission to collect the samples of P. chienii, and Motuo National Nature Reserve, who provided permission to collect the samples of T. yunnanensis. The formal identification of the plant material was undertaken by Dr. Lei Zhang (Washington State University). A voucher specimen of this material has not been deposited in a publicly available herbarium. Experimental researches on Tauxs trees comply with Hangzhou Normal university guidelines. For metabolomic analysis, fresh twigs were harvested from 10 independent trees of $P$. chienii and $T$. yunnanensis, respectively. For transcriptomic analysis, fresh twigs were harvested from 3 independent trees of P. chienii and T. yunnanensis, respectively. All the samples were ground in liquid nitrogen and transferred to a tube.

\section{Metabolite extraction and sample preparation}

Each sample was added mixed with an aliquot of $500 \mu \mathrm{L}$ pre-colded 50\% methanol with several steel balls. The mixture solution was shaken at a rate of 1900 strokes/ min for 2 min using a 2010 Geno/Grinder (SPEX SamplePrep, Metuchen, NJ, USA). After centrifugation at $4000 \mathrm{~g}$ for $20 \mathrm{~min}$, the supernatants were transferred into new 96 -well plates. The samples were stored at $-80^{\circ} \mathrm{C}$ prior to the UPLC-MS/MS analysis. In addition, the quality control samples were also prepared by combining $10 \mu \mathrm{L}$ of each extraction mixture.

\section{Untargeted metabolomic profiling}

All samples were analyzed by the UPLC-MS/MS system according to our previous work [32]. Firstly, all chromatographic separations were performed using an ultra performance liquid chromatography (UPLC) system (SCIEX, UK). An ACQUITY UPLC BEH Amide column $(100 \mathrm{~mm} \times 2.1 \mathrm{~mm}, 1.7 \mu \mathrm{m}$, Waters, Milford, MA, USA) was used for the reversed phase separation. The column oven was maintained at $35^{\circ} \mathrm{C}$. The flow rate was $0.4 \mathrm{~mL} /$ $\mathrm{min}$ and the mobile phase consisted of solvent $\mathrm{A}(25$ $\mathrm{mM}$ ammonium acetate and $25 \mathrm{mM} \mathrm{NH}_{4} \mathrm{H}_{2} \mathrm{O}$ ) and solvent $\mathrm{B}$ (IPA:CAN $=9: 1, \mathrm{v} / \mathrm{v}$, and $0.1 \%$ of formic acid). 
Gradient elution conditions were set as follows: $0 \sim 0.5$ min, $95 \%$ of solvent B; $0.5 \sim 9.5 \mathrm{~min}, 95$ to $65 \%$ of solvent B; $9.5 \sim 10.5 \mathrm{~min}, 65 \% \sim 40 \%$ of solvent $\mathrm{B} ; 10.5 \sim$ $12 \mathrm{~min}, 40 \%$ of solvent B; $12 \sim 12.2 \mathrm{~min}, 40 \% \sim 95 \%$ of solvent B; $12.2 \sim 15 \mathrm{~min}, 95 \%$ of solvent $\mathrm{B}$. The injection volume for each sample was set at $4 \mu \mathrm{L}$.

A high-resolution MS/MS TripleTOF 5600 plus (SCIEX, UK) was used to recognize the metabolites eluted from the column. The TOF was carried out in both positive and negative ion modes. The detail parameters of UPLC-MS/MS analysis were set according to our previous work [32]. Furthermore, in order to evaluate the stability of the UPLC-MS/MS system during the whole data acquisition process, one quality control sample was detected after every 10 samples.

\section{Bioinformatics of the metabolomic datasets}

The acquired MS data features, including peak picking, peak grouping, retention time (RT), second peak grouping, and annotation of isotopes and adducts was performed using XCMS software [57]. Each ion was identified by combining $\mathrm{RT}$ and $\mathrm{m} / \mathrm{z}$ data together. Intensities of each peak were recorded and a $3 \mathrm{D}$ matrix, containing each assigned peak indexs (retention time-m/ $\mathrm{z}$ pair), sample name and ion intensity, was generated.

Metabolites were annotated by matching the exact $\mathrm{m}$ / $\mathrm{z}$ of samples with those from the online KEGG and PLANTCYC database. The molecular formulas of all annoated metabolites were further validated by the isotopic distribution measurements. The intensity of peak data was further preprocessed by an in-house software metaX. Low quality features that were detected in less than $50 \%$ of quality control samples or $80 \%$ of experiment samples were removed. The high quatily peaks were imputed with the k-nearest neighbor algorithm to further improve their quality. PCA was performed for outlier detection and batch effects evaluation using the pre-processed dataset. In addition, the relative standard deviations (SD) of the metabolic features were calculated across all quality control samples to remove the features with SD > 30\%.

To screen differential accumulated metabolites, the default setting parameters, such as VIP $>1, P<0.05$, were used. The statistical analyses in metabolomics, including univariate analysis and multivariate PLS-DA analysis with corresponding VIP value, were performed. The univariate analysis is Wilcoxon test with $P$ value corrected by $\mathrm{BH}$ correction.

\section{RNA extraction and sequencing}

Total RNAs were extracted using an RNeasy plant mini kit (Qiagen, Hilden, Germany) according to its manual. Then, $10 \mu \mathrm{g}$ of each RNA sample (three biological replicates) was used for library construction. cDNA library construction was performed according to the method previously described by $\mathrm{Yu}$ et al. [30]. Sequencing was performed using an Illumina Hiseq 4000 platform (LCBio, Hangzhou, China) according to its protocol.

Raw RNA-seq reads were trimmed for low quality reads with length shorter than $25 \mathrm{bp}$. Based on the clean reads, transcriptomic assembly was perfromed using Trinity software [58]. The raw sequence data have been submitted to the NCBI Short Read Archive with accession number GSE121523 and GSE121831.

\section{Analysis of the transcriptomic datasets}

For gene annotation, all assembled sequences were searched against various databases, such as nonredundant (Nr) protein, Gene Ontology (GO), SwissProt, and Kyoto Encyclopedia of Genes and Genomes (KEGG) databases. We used the blastx function in dismond software to search the assembled genome against with different databases, setting evalue $<0.00001$. When a gene sequence could be aligned to multiple protein, the result with minimun evalue were selected as the final annotation of the gene. According to the protein ID, the conresponding gene symbol is obtained.

Expression levels for the unigenes were calculated by transcripts per million method. The transcript abundance values of each unigene in different sample groups were transformed into Z-score after log transformation. The differentially expressed genes (DEGs) were screened with criterions: $\log 2$ (fold change of transcript abundance) $>1$ and statistical significance $P<0.05$. GO and KEGG enrichment analysis of the DEGs were performed on the DEGs by perl scripts in house. The heatmap was made using MultiExperiment Viewer (version 4.9.0). Software samtools was used to analyze and filter the SNP with default parameters. All SNPs with mindepth less than 100 were removed.

\section{Quantificative analysis of targeted taxoids}

Fresh twigs were collected from six independent trees of each species. The samples were thoroughly dried at $40{ }^{\circ} \mathrm{C}$ and then ground into fine powder. A modified version of a previously published method was used to prepare crude extracts [30]. Six common taxoids, including 10-deacetylbaccatin III (10-DAB), baccatin III (BAC), 10-deacetylpaclitaxel (DAP), paclitaxel (PTX), 7-epi 10desacetyl paclitaxel (7-E-DAP) and 7-epipaclitaxel (7-EPTX) were quantified using a UPLC-MS/MS method. Before UPLC-MS/MS analysis, the crude extracts were diluted at a ratio of $1: 10$ and were passed through $0.22 \mu \mathrm{m}$ membrane filters.

For the separation of these taxoids, a Kinetex $\mathrm{C}_{18}$ column $(100 \times 4.6 \mathrm{~mm}, 2.6 \mu \mathrm{m}$, Phenomenex, Torrance, CA, USA) was used. The determination of these taxoids was performed using a LC-30 AD UPLC (Shimadzu, 
Japan) coupled with a SCIEX QTRAP 6500 MS (Applied Biosystems). The Multi-Quant software (version 3.0) was applied for data acquisition and processing. Multiple reaction monitoring (MRM) was used in the positive ionization mode. The transitions of $\mathrm{m} / z \quad 567.4 \rightarrow 445.3$ for 10 -DAB, $m / z \quad 609.5 \rightarrow 427.3$ for BAC, $m / z 834.4 \rightarrow$ 308.2 for DAP, $m / z \quad 876.4 \rightarrow 308.2$ for PTX, $m / z$ $876.4 \rightarrow 591.4$ for 7 -E-PTX, and $\mathrm{m} / z \quad 834.4 \rightarrow 308.2$ for 7-E-DAP were used for the quantification. The quantification of seven taxoids was carried out using an LC-30 AD UPLC system (Shimadzu, Japan) coupled with a SCIEX QTRAP 6500 mass spectrometer (Applied Biosystems). The results were presented as the means of at least three replicates \pm standard errors.

\section{Real-time PCR validation}

Real-time PCR experiment was performed according to our previous work [30]. In brief, the SYBR Premix Ex Taq Kit (TaKaRa, Dalian, China) and a DNA Sequence Detection System (ABI PRIM 7700) were used. Independent cDNA samples from $P$. chienii and T. yunnanensis were used for real-time PCR experiments. An ACTIN sequence was used as the internal standard gene to calculate relative fold differences by the values of comparative cycle threshold $\left(2^{-\Delta \Delta C t}\right)$. The expression analysis was performed for three biological replications. The primer sequences were listed in Additional file 12.

\section{Statistical analysis}

Statistical analyses were carried out using SPSS software version 19.0 (SPSS Inc., Chicago, IL, USA), and a oneway ANOVA was applied to compare the differences in taxoid contents between the two sample groups. Wilcoxon tests were conducted to detect differences in metabolite concentrations between two species. The $P$ value was adjusted for multiple tests using an FDR (Benjamini-Hochberg). Supervised PLS-DA was conducted through metaX to discriminate the different variables between two groups. The VIP value was calculated. A VIP cut-off value of 1.0 was used to select important features.

\section{Supplementary Information}

The online version contains supplementary material available at https://doi. org/10.1186/s12870-021-02883-0.

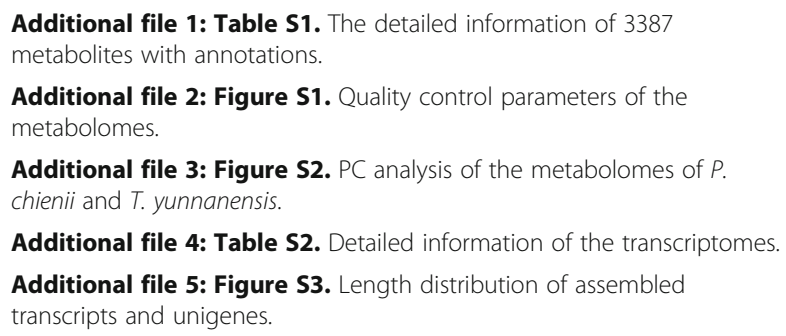

Additional file 6: Table S3. GO categories of all the DEGs between $T$. yunnanensis and $P$. chienii.

Additional file 7: Table S4. KEGG enriched analysis of the DEGS between $T$. yunnanensis and $P$. chienii.

Additional file 8: Table S5. Significance values of each KEGG term belonging to primary and secondary metabolism.

Additional file 9: Table S6. The detailed information of the unigenes encoding the enzymes that are involved in the MEP pathway.

Additional file 10: Table S7. The detailed information of the unigenes encoding the enzymes that are involved in the taxol biosynthesis pathway.

Additional file 11: Table S8. The number of identified TFs and differentially expressed TFs.

Additional file 12: Table S9. The primer sequences for qRT-PCR.

\section{Abbreviations}

GGPP: Geranylgeranyl diphosphate; IPP: Isopentenyl diphosphate; DMAPP: Dimethylallyl diphosphate; MEP: 2-C-methyl-D-erythritol phosphate; TS: Taxadiene synthase; 10-DAB: 10-deacetylbaccatin-III; 10-DAB III: 10deacetylbaccatin III; HPLC-MS/MS: High-performance liquid chromatographytandem mass spectrometry; ANOVA: Analysis of variance; PCA: Principal component analysis; DAM: Differential accumulated metabolite

\section{Acknowledgements}

We are also grateful to LC Sciences company (Hangzhou, China) for technical support. We are grateful to Kaidong Liu (Life Science and Technology School, Lingnan Normal University) for reading this manuscript.

\section{Authors' contributions}

CS and HW made substantial contributions to conception, was involved in drafting the manuscript, and given final approval of the version to be published. CY, XL, CZ and XX made substantial contributions to acquisition of data analysis, was involved in revising the manuscript, and given final approval of the version to be published. $\mathrm{CY}, \mathrm{XL}, \mathrm{JH}$ and $\mathrm{YC}$ made substantial contributions to analysis and interpretation of data, was involved in revising the manuscript, and given final approval of the version to be published. All authors have read and approved the manuscript, and ensure that this is the case.

\section{Funding}

This work was funded by Zhejiang Provincial Natural Science Foundation of China under Grant No. LY19C160001; Open Foundation of State Key Laboratory of Subtropical Silviculture, Zhejiang A \& F University (KF201708); Major Increase Or Decrease Program In The Central Finance Level (Grant No. 2060302); and Zhejiang Provincial key Research \& Development Project Grants (2017C02011, 2018C02030). There is no role of the funding body in the design of the study and collection, analysis, and interpretation of data and in writing the manuscript.

\section{Availability of data and materials}

The raw sequence data has been submitted to the NCBI Short Read Archive with accession numbers GSE121523 and GSE121831.

\section{Ethics approval and consent to participate}

This project uses plant materials and does not utilize transgenic technology. We complied with all relevant institutional, national and international guidelines and the appropriate permissions were obtained from the Mount Tianmu National Nature Reserve and Motuo National Nature Reserve. The authorities responsible for the Taxus resources are the Mount Tianmu National Nature Reserve, who provided permission to collect the samples of P. chienii, and Motuo National Nature Reserve, who provided permission to collect the samples of T. yunnanensis, for our scientific research.

\section{Consent for publication}

Not applicable.

\section{Competing interests}

The authors declare that they have no competing interests. 


\section{Author details}

'College of Life and Environmental Sciences, Hangzhou Normal University, Hangzhou 310036, China. ${ }^{2}$ Zhejiang Provincial Key Laboratory for Genetic Improvement and Quality Control of Medicinal Plants, Hangzhou Normal University, Hangzhou 310036, China.

Received: 3 September 2020 Accepted: 9 February 2021

Published online: 19 February 2021

\section{References}

1. Fu Y, Li S, Zu Y, Yang G, Yang Z, Luo M, et al. Medicinal chemistry of paclitaxel and its analogues. Curr Med Chem. 2009;16(30):3966-85.

2. Zhou T, Luo X, Yu C, Zhang C, Zhang L, Song Y-B, et al. Transcriptome analyses provide insights into the expression pattern and sequence similarity of several taxol biosynthesis-related genes in three Taxus species. BMC Plant Biol. 2019;19(1):33.

3. Li Y, Qin F, Wang SM, Guo RX, Zhang YF, Gu YC, et al. Chemical studies on Taxus canadensis. Chem Biodivers. 2013;10(10):1729-53.

4. Li ST, Zhang P, Zhang M, Fu CH, Zhao CF, Dong YS, et al. Transcriptional profile of Taxus chinensis cells in response to methyl jasmonate. BMC Genomics. 2012;13:295

5. Deng Q, Zhang H, He Y, Wang T, Su Y. Chloroplast microsatellite markers for Pseudotaxus chienii developed from the whole chloroplast genome of Taxus chinensis var. mairei (Taxaceae). Appl Plant Sci. 2017; 5(3):apps.1600153.

6. Dorken VM, Nimsch H, Rudall PJ. Origin of the Taxaceae aril: evolutionary implications of seed-cone teratologies in Pseudotaxus chienii. Ann Bot. 2019; 123(1):133-43

7. Hsu C-Y, Wu C-S, Chaw S-M. Ancient nuclear plastid DNA in the yew family (Taxaceae). Genome Biol Evol. 2014;6(8):2111-21.

8. Sanchez-Munoz R, Bonfill M, Cusido RM, Palazon J, Moyano E. Advances in the regulation of in vitro paclitaxel production: methylation of a Y-patch promoter region alters BAPT gene expression in Taxus cell cultures. Plant Cell Physiol. 2018;59(11):2255-67.

9. Croteau R, Ketchum RE, Long RM, Kaspera R, Wildung MR. Taxo biosynthesis and molecular genetics. Phytochem Rev. 2006;5(1):75-97.

10. Appendino G. The phytochemistry of the yew tree. Nat Prod Rep. 1995; 12(4):349-60.

11. Yang L, Zheng ZS, Cheng F, Ruan X, Jiang DA, Pan CD, et al. Seasona dynamics of metabolites in needles of Taxus wallichiana var. mairei. Molecules. 2016;21(10):1403.

12. Siegle $L$, Pietsch J. Taxus ingredients in the red arils of Taxus baccata $L$. determined by HPLC-MS/MS. Phytochem Anal. 2018;29(5):446-51.

13. Wang $\mathrm{Y}$, Wang J, Wang $\mathrm{H}$, Ye W. Novel taxane derivatives from Taxus wallichiana with high anticancer potency on tumor cells. Chem Biol Drug Des. 2016;88(4):556-61.

14. Liu HS, Gao YH, Liu LH, Liu W, Shi QW, Dong M, et al. Inhibitory effect of 13 taxane diterpenoids from Chinese yew (Taxus chinensis var. mairei) on the proliferation of HeLa cervical cancer cells. Biosci Biotechnol Biochem. 2016; 80(10):1883-6

15. Slichenmyer WJ, Von Hoff DD. New natural products in cancer chemotherapy. J Clin Pharmacol. 1990;30(9):770-88,

16. Cao W, Liu Y, Zhang R, Zhang B, Wang T, Zhu X, et al. Homoharringtonine induces apoptosis and inhibits STAT3 via IL-6/JAK1/STAT3 signal pathway in Gefitinib-resistant lung cancer cells. Sci Rep. 2015;5:8477.

17. Wang YF, Shi QW, Dong M, Kiyota H, Gu YC, Cong B. Natural taxanes: developments since 1828. Chem Rev. 2011;111(12):7652-709.

18. Ansbacher T, Freud Y, Major DT. Slow-starter enzymes: role of active-site architecture in the catalytic control of the biosynthesis of taxadiene by taxadiene synthase. Biochemistry. 2018;57(26):3773-9.

19. D'Auria JC. Acyltransferases in plants: a good time to be BAHD. Curr Opin Plant Biol. 2006;9(3):331-40

20. Kaspera R, Croteau R. Cytochrome P450 oxygenases of Taxol biosynthesis. Phytochem Rev. 2006; (2-3):433-44.

21. Walker K, Croteau R. Taxol biosynthesis: molecular cloning of a benzoyl-CoA: taxane 2alpha-O-benzoyltransferase CDNA from Taxus and functional expression in Escherichia coli. Proc Natl Acad Sci U S A. 2000:97(25):13591-6.

22. Jiang M, Stephanopoulos G, Pfeifer BA. Downstream reactions and engineering in the microbially reconstituted pathway for Taxol. Appl Microbiol Biotechnol. 2012;94(4):841-9.
23. Walker K, Long R, Croteau R. The final acylation step in taxol biosynthesis: cloning of the taxoid C13-side-chain N-benzoyltransferase from Taxus. Proc Natl Acad Sci U S A. 2002;99(14):9166-71.

24. Zhou X, Zhong JJ. Quantitative influence of endogenous salicylic acid level on taxuyunnanine $\mathrm{C}$ biosynthesis in suspension cultures of Taxus chinensis. Biotechnol Bioeng. 2011;108(1):216-21.

25. Kochkin DV, Globa EB, Demidova EV, Gaisinsky W, Galishev BA, Kolotyrkina $\mathrm{NG}$, et al. Occurrence of 14-hydroxylated taxoids in the plant in vitro cell cultures of different yew species (Taxus spp.). Dokl Biochem Biophys. 2017; 476(1):337-9.

26. Ketchum RE, Rithner CD, Qiu D, Kim YS, Williams RM, Croteau RB. Taxus metabolomics: methyl jasmonate preferentially induces production of taxoids oxygenated at C-13 in Taxus $\times$ media cell cultures. Phytochemistry. 2003;62(6):901-9.

27. Miao YC, Lang XD, Zhang ZZ, Su JR. Phylogeography and genetic effects of habitat fragmentation on endangered Taxus yunnanensis in Southwest China as revealed by microsatellite data. Plant Biol. 2014;16(2):365-74

28. Hai P, Wen SZ, Li Y, Gao Y, Jiang XJ, Wang F. New taxane diterpenoids from Taxus yunnanensis. Nat Prod Bioprospect. 2014;4(1):47-51.

29. Yan C, Yin Y, Zhang D, Yang W, Yu R. Structural characterization and in vitro antitumor activity of a novel polysaccharide from Taxus yunnanensis. Carbohydr Polym. 2013;96(2):389-95.

30. Yu C, Guo H, Zhang Y, Song Y, Pi E, Yu C, et al. Identification of potential genes that contributed to the variation in the taxoid contents between two Taxus species (Taxus media and Taxus mairei). Tree Physiol. 2017;37(12): 1659-71.

31. Hao J, Guo H, Shi X, Wang Y, Wan Q, Song YB, et al. Comparative proteomic analyses of two Taxus species (Taxus $\times$ media and Taxus mairei) reveals variations in the metabolisms associated with paclitaxel and other metabolites. Plant Cell Physiol. 2017:58(11):1878-90.

32. Yu C, Luo X, Zhan X, Hao J, Zhang L, Song YBL, et al. Comparative metabolomics reveals the metabolic variations between two endangered Taxus species (T. fuana and T. yunnanensis) in the Himalayas. BMC Plant Biol. 2018;18(1):197.

33. He CT, Li ZL, Zhou Q, Shen C, Huang YY, Mubeen S, et al. Transcriptome profiling reveals specific patterns of paclitaxel synthesis in a new Taxus yunnanensis cultivar. Plant Physiol Biochem. 2018;122:10-8.

34. Mubeen S, Li ZL, Huang QM, He CT, Yang ZY. Comparative transcriptome analysis revealed the tissue-specific accumulations of taxanes among yhree experimental lines of Taxus yunnanensis. J Agric Food Chem. 2018;66(40): 10410-20.

35. Eisenreich W, Menhard B, Hylands PJ, Zenk MH, Bacher A. Studies on the biosynthesis of taxol: the taxane carbon skeleton is not of mevalonoid origin. Proc Natl Acad Sci U S A. 1996;93(13):6431-6.

36. John Goodman W. V: the story of taxol: science and politics in the making of an anticancer drug; 2001.

37. Vranova E, Coman D, Gruissem W. Network analysis of the MVA and MEP pathways for isoprenoid synthesis. Annu Rev Plant Biol. 2013;64:665-700.

38. Ketchum RE, Gibson DM, Croteau RB, Shuler ML. The kinetics of taxoid accumulation in cell suspension cultures of Taxus following elicitation with methyl jasmonate. Biotechnol Bioeng. 1999:62(1):97-105.

39. Estevez JM, Cantero A, Reindl A, Reichler S, Leon P. 1-Deoxy-D-xylulose-5phosphate synthase, a limiting enzyme for plastidic isoprenoid biosynthesis in plants. J Biol Chem. 2001;276(25):22901-9.

40. Laskaris G, Jong CFD, Jaziri M, Heijden RVD, Theodoridis G, Verpoorte R. Geranylgeranyl diphosphate synthase activity and taxane production in Taxus baccata cells. Phytochemistry. 1999;50(50):939-46.

41. Soliman SSM, Mosa KA, El-Keblawy AA, Husseiny MI. Exogenous and endogenous increase in fungal GGPP increased fungal Taxol production. Appl Microbiol Biotechnol. 2017;101(20):7523-33.

42. Escorcia AM, van Rijn JPM, Cheng GJ, Schrepfer P, Bruck TB, Thiel W. Molecular dynamics study of taxadiene synthase catalysis. J Comput Chem. 2018;39(19):1215-25

43. Walker K, Ketchum RE, Hezari M, Gatfield D, Goleniowski M, Barthol A, et al. Partial purification and characterization of acetyl coenzyme a: taxa-4(20),11(12)-dien-5alpha-ol O-acetyl transferase that catalyzes the first acylation step of taxol biosynthesis. Arch Biochem Biophys. 1999; 364(2):273-9.

44. You LF, Wei T, Zheng QW, Lin JF, Guo LQ, Jiang BH, et al. Activity essentia residue analysis of taxoid 10beta-O-acetyl transferase for enzymatic synthesis of baccatin. Appl Biochem Biotechnol. 2018;186(4):949-59. 
45. Walker K, Fujisaki S, Long R, Croteau R. Molecular cloning and heterologous expression of the $\mathrm{C}-13$ phenylpropanoid side chain-CoA acyltransferase that functions in Taxol biosynthesis. Proc Natl Acad Sci U S A. 2002;99(20):12715-20.

46. Tatini LK, Rao NS, Khan M, Peraka KS, Reddy KV. Concomitant pseudopolymorphs of 10-deacetyl baccatin III. AAPS PharmSciTech. 2013; 14(2):558-68.

47. Kupeli E, Erdemoglu N, Yesilada E, Sener B. Anti-inflammatory and antinociceptive activity of taxoids and lignans from the heartwood of Taxus baccata L. J Ethnopharmacol. 2003;89(2-3):265-70.

48. Kuang X, Sun S, Wei J, Li Y, Sun C. Iso-Seq analysis of the Taxus cuspidata transcriptome reveals the complexity of Taxol biosynthesis. BMC Plant Biol. 2019;19(1):210

49. Lenka SK, Nims NE, Vongpaseuth K, Boshar RA, Roberts SC, Walker EL. Jasmonate-responsive expression of paclitaxel biosynthesis genes in Taxus cuspidata cultured cells is negatively regulated by the bHLH transcription factors TCJAMYC1, TCJAMYC2, and TCJAMYC4. Front Plant Sci. 2015;6:115.

50. Zhang M, Li S, Nie L, Chen Q, Xu X, Yu L, et al. Two jasmonateresponsive factors, TCERF12 and TCERF15, respectively act as repressor and activator of tasy gene of taxol biosynthesis in Taxus chinensis. Plant Mol Biol. 2015;89(4-5):463-73.

51. Zhang M, Chen Y, Nie L, Jin X, Liao W, Zhao S, et al. Transcriptome-wide identification and screening of WRKY factors involved in the regulation of taxol biosynthesis in Taxus chinensis. Sci Rep. 2018;8(1):5197.

52. Yanfang $Y$, Kaikai Z, Liying Y, Xing L, Ying W, Hongwei L, et al. Identification and characterization of MYC transcription factors in Taxus sp. Gene. 2018; 675:1-8.

53. Cui Y, Mao R, Chen J, Guo Z. Regulation mechanism of MYC family transcription factors in jasmonic acid signalling pathway on taxol biosynthesis. Int J Mol Sci. 2019;20(8):1843.

54. Zhang M, Jin X, Chen Y, Wei M, Liao W, Zhao S, et al. TCMYC2a, a basic helix-loop-helix transcription factor, transduces JA-signals and regulates taxol biosynthesis in Taxus chinensis. Front Plant Sci. 2018;9:863.

55. Yu C, Luo X, Zhang C, Xu X, Huang J, Chen Y, et al. Tissue-specific study across the stem of Taxus media identifies a phloem-specific TmMYB3 involved in the transcriptional regulation of paclitaxel biosynthesis. Plant J. 2020;103(1):95-110.

56. Hu X, Zhang L, Wilson I, Shao F, Qiu D. The R2R3-MYB transcription factor family in Taxus chinensis: identification, characterization, expression profiling and posttranscriptional regulation analysis. PeerJ. 2020;8:e8473.

57. Smith CA, Want EJ, O'Maille G, Abagyan R, Siuzdak G. XCMS: processing mass spectrometry data for metabolite profiling using nonlinear peak alignment, matching, and identification. Anal Chem. 2006;78(3):779-87.

58. Grabherr MG, Haas BJ, Yassour M, Levin JZ, Thompson DA, Amit I, et al. Trinity: reconstructing a full-length transcriptome without a genome from RNA-Seq data. Nat Biotechnol. 2011;29(7):644-52.

\section{Publisher's Note}

Springer Nature remains neutral with regard to jurisdictional claims in published maps and institutional affiliations.

\section{Ready to submit your research? Choose BMC and benefit from}

- fast, convenient online submission

- thorough peer review by experienced researchers in your field

- rapid publication on acceptance

- support for research data, including large and complex data types

- gold Open Access which fosters wider collaboration and increased citations

- maximum visibility for your research: over $100 \mathrm{M}$ website views per year

At BMC, research is always in progress.

Learn more biomedcentral.com/submissions 